Rodrigo Cauduro Roscani (iD) http://orcid.org/0000-0001-9113-2380

Paulo Alves Maia ${ }^{\mathrm{a}}$

iD https://orcid.org/0000-0002-6089-892X

Maria Inês Monteiro ${ }^{b}$

iD https://orcid.org/0000-0002-6004-8378

${ }^{a}$ Fundação Jorge Duprat Figueiredo de Segurança e Medicina do Trabalho (Fundacentro), Escritório de Representação em Campinas. Campinas, SP, Brasil.

b Universidade Estadual de Campinas (Unicamp), Faculdade de Enfermagem. Campinas, SP, Brasil.

Contato:

Rodrigo Cauduro Roscani

E-mail:

rodrigo.roscani@fundacentro.gov.br

Os autores declaram que o trabalho não foi subvencionado e que não há conflitos de interesses.

Os autores informam que o trabalho não foi baseado em tese ou dissertação e foi apresentado no $2^{\circ}$ Seminário Nacional sobre Saúde e Trabalho: contribuições da saúde do trabalhador para o trabalho decente (Ribeirão Preto, 2017), com publicação do resumo nos anais do evento.

\section{Sobrecarga térmica em áreas rurais: a influência da intensidade do trabalho}

\author{
Thermal overload in rural areas: the influence of work intensity
}

\section{Resumo}

Introdução: a legislação brasileira determina que se monitore o Índice de Bulbo Úmido Termômetro de Globo (IBUTG) nos ambientes de trabalho em que o calor possa representar risco à saúde dos trabalhadores. Porém, esse monitoramento e a adoção de medidas preventivas não são comumente observados. Objetivo: identificar e mapear a influência da intensidade do trabalho na extrapolação potencial de limites de sobrecarga térmica para trabalhadores em atividade a céu aberto em áreas rurais do Nordeste do Brasil. Métodos: com base na Norma Regulamentadora $\mathrm{n}^{\mathrm{O}} 15$ e em dados do Instituto Nacional de Meteorologia, o software Sobrecarga Térmica foi utilizado para estimar os índices de IBUTG na região, no período de $1^{\circ}$ de setembro de 2016 a 31 de agosto de 2017. Resultados: dos 132 pontos geográficos investigados, a extrapolação dos limites de sobrecarga térmica foi identificada em $48 \%$ dos trabalhos pesados e em $14 \%$ dos trabalhos moderados, indicando a necessidade de paradas para descanso em mais de $50 \%$ do tempo analisado. Discussão: a diminuição da intensidade do trabalho resulta em redução expressiva da sobrecarga térmica. Nas localidades historicamente reconhecidas pelo alto risco de exposição ao calor, deveria ser adotada, como medida efetiva de prevenção, a restrição ao estímulo de atividades extenuantes, sobretudo as promovidas pela remuneração atrelada à produtividade.

Palavras-chave: saúde do trabalhador; exaustão por calor; prevenção primária; trabalho a céu aberto.

\begin{abstract}
Introduction: Brazilian law requires the use of Wet-Bulb Globe Temperature (WBGT) to monitor work environments where heat may pose a risk to workers' health; however, such monitoring is often not observed and preventive measures are not taken. Objective: to identify and map the influence of labor intensity on the potential extrapolation of heat exposure threshold limits for outdoor workers in rural areas of northeastern Brazil. Methods: based on Brazil's Labor Regulatory Standard no. 15 and data from the Brazilian Institute of Meteorology, the software Sobrecarga Térmica (Thermal Overload) was used to estimate WBGT index in the territory from September 1st, 2016 to August 31, 2017. Results: the extrapolation of thermal overload limits was characterized for heavy work in $48 \%$, and for moderate work in $14 \%$ of the 132 geographic points investigated, indicating the need for rest stops in more than $50 \%$ of the analyzed period. Discussion: decreasing work intensity results in an expressive reduction in thermal overload rates. The restriction to the stimulation of strenuous activities - especially remunerated ones linked to production - in regions historically recognized for their high risk of heat exposure should be implemented as an effective preventive measure for heat stress.
\end{abstract}

Keywords: occupational health; heat stroke; primary prevention; outdoor work. 


\section{Introdução}

Pesquisas mostram que o aumento do calor está associado ao crescimen to da taxa de morbidade e mortalidade. Um estudo analisou um período de 42 anos na França e apontou forte correlação entre as curvas de temperatura e mortalidade ${ }^{1}$. Uma revisão sobre o aspecto epidemiológico da mortalidade relacionada ao calor reuniu evidências mostrando que exposições ao calor intenso por mais de 3 semanas provocam significativos aumentos nas taxas de mortalidade, especialmente para faixas etárias mais avançadas ${ }^{2}$. Atividades físicas extenuantes e o uso de roupas e equipamentos de proteção que dificultam a troca de calor com o ambiente potencializam o risco de sobrecarga térmica pela interferência na regulação térmica corporal, com elevação da temperatura interna do indivíduo a níveis prejudiciais à saúde ${ }^{3}$.

Os efeitos do calor sobre o trabalhador são estudados de forma ampla, em várias regiões do mundo e em diferentes atividades laborais. Complicações renais foram relatadas na Tailândia, principalmente em trabalhadores rurais ${ }^{4}$, e consequências cardiovasculares foram descritas em cortadores de cana-de-açúcar no Brasil ${ }^{5,6}$. Em trabalhadores de fazendas do estado do Oregon, nos EUA, foram descritos diversos sintomas relacionados ao calor, como erupções cutâneas, cãibras, espasmos musculares, tontura, desmaio, dor de cabeça, sudorese severa, fadiga e extrema fraqueza, náuseas, vômito e estado de confusão ${ }^{7}$. Há ainda registros de casos extremos no estado da Carolina do Norte, EUA, entre 1977 e 2001, onde foram relatadas 40 mortes relacionadas ao calor no local de trabalho, das quais 16 ocorreram em propriedades rurais ${ }^{8}$. Também em ambientes industriais fechados, no Canadá, foram identificados trabalhadores com disfunções cognitivas em consequência do calor no ambiente de trabalho ${ }^{9}$.

A legislação brasileira vigente, no Anexo $\mathrm{n}^{\circ} 3$ ("Limites de tolerância para exposição ao calor") da Norma Regulamentadora $\mathrm{n}^{\circ}$ 15: atividades e operações insalubres $(\mathrm{NR} 15)^{\mathrm{C}}$, do Ministério do Trabalho, preconiza limites do Índice de Bulbo Úmido Termômetro de Globo (IBUTG) como parâmetro de análise para a quantificação do risco de sobrecarga térmica de acordo com o regime de trabalho e o tipo de atividade realizados ${ }^{10}$. Os tipos de atividade física são classificados em leve, moderado ou pesado, de acordo com o trabalho realizado. O IBUTG foi inicialmente desenvolvido para estudar as relações entre o calor e suas consequências fisiológicas durante treinamentos militares ${ }^{11}$. O índice é descrito pela norma ISO $7243^{12}$, e sua utilização na análise de sobrecarga térmica laboral é usual em estudos de diversas regiões do mundo ${ }^{13,14}$.

A NR15 ${ }^{10}$ e a Norma Regulamentadora $n^{\circ} 9$ Programa de Prevenção de Riscos Ambientais $\left({ }^{N R 9}\right)^{15}$ determinam que as medições do IBUTG sejam feitas no local em que o trabalhador executa suas atividades. Entretanto, para os casos de atividades a céu aberto, quando se trata de áreas com grandes extensões ou elevado número de trabalhadores - o que é comum nas áreas rurais -, podem ocorrer dificuldades financeiras e operacionais para monitoramento do calor, como falta de pessoal técnico. Nesse sentido, torna-se importante a utilização de métodos alternativos de monitoramento do calor, como o software Sobrecarga Térmica ${ }^{16}$, que estima o IBUTG a partir de variáveis meteorológicas observadas pelas redes automáticas do Instituto Nacional de Meteorologia (Inmet).

Considerando que no Brasil não se monitora diariamente o IBUTG nas atividades rurais, ou seja, que a dimensão do risco não é conhecida, pode-se afirmar que as medidas preventivas, quando aplicadas, são ineficazes contra as doenças provocadas pela exposição excessiva ao calor. Além disso, o adoecimento dos trabalhadores pelo calor é desconhecido pelas estatísticas oficiais, seja em suas manifestações de formas agudas, como cãibras ou desmaios ${ }^{7}$ e até morte ${ }^{8}$, ou de formas crônicas, como comprometimento renal ${ }^{4}$ ou cardiovascular ${ }^{5,6}$.

Há, contudo, localidades nas quais o calor ambiental notoriamente extrapola os limites. Tal caracterização pode ser feita pela análise histórica do clima nessas regiões. Nessas condições, estratégias de prevenção deveriam ser tomadas de antemão, sem a necessidade ou dependência do monitoramento em tempo real.

É importante destacar que o IBUTG, por ter origem em práticas de prevenção de sobrecarga térmica em treinamentos militares ${ }^{11}$, se baseia em características de atividades bem definidas, além de que os militares em treinamento têm condições físicas e alimentares controladas. Isso não ocorre em relação aos trabalhadores rurais, que de modo geral exercem suas atividades sob condições sanitárias, ambientais e alimentares precárias. Dessa forma, os limites estabelecidos com base no IBUTG podem estar superestimando a resistência desses trabalhadores à exposição ao calor.

c A NR15 e seus anexos estão passando por revisão, em um processo de atualização que foi lançado pelo Governo Federal em 30 de junho de 2019. 
Ressalta-se que é comum prevalecer a prática de priorizar a produtividade em detrimento da segurança e da saúde do trabalhador. Os próprios trabalhadores, mesmo conscientes dos riscos e apresentando sintomas de agravos à saúde, como cãibras e tonturas, por exemplo, aderem às metas de produtividade quando "motivados" por pagamento diferenciado em função da produção individual $^{5}$. Um estudo sobre exposição de trabalhadores rurais ao calor em países de média renda identificou que o aumento do risco de sobrecarga térmica está relacionado ao aumento do ritmo de trabalho, incentivado pelo pagamento diferenciado da produção ${ }^{3}$. Ilustrando esse cenário, de maneira particular para o cortador de cana-de-açúcar, dados mostram que a produtividade média diária dessa cultura na década de 1950 era de 3 toneladas, passando para 6 na década de 1980, e 12 no final da década de $1990^{17}$ - um incremento de produtividade de $300 \%$. Também se observou, por parte de empregadores, o estabelecimento de metas de corte mínimas de 10 toneladas diárias, ocasionando, como consequência dos níveis de exaustão, a dispensa de cerca de $40 \%$ de uma turma de cortadores no primeiro mês de trabalho e de mais $20 \%$ no segundo mês, permanecendo na atividade apenas os que apresentavam maior produtividade ${ }^{18}$.

Além dos aspectos relativos ao trabalho propriamente dito, em perspectiva futura, de maneira global, espera-se o agravamento das condições de risco relacionadas à sobrecarga térmica dos trabalhadores a céu aberto em função das mudanças climáticas que vêm sendo apontadas por diversos estudos e reconhecidas pela Organização das Nações Unidas (ONU) ${ }^{19}$.

Segundo a Organização Meteorológica Mundial $(\mathrm{OMM})^{19}$, em 2016 as temperaturas médias globais fixaram novo recorde, e o mundo testemunhou o degelo marinho, o aumento do nível do mar e do calor oceânico. De acordo com a agência, as temperaturas globais atingiram $1,1^{\circ} \mathrm{C}$ acima da média do período pré-industrial, com maior crescimento a partir da década de 1950, registrando-se um incremento, em valores absolutos, de $0,9394^{\circ} \mathrm{C}$, considerando as temperaturas de superfície de continentes e oceanos. Se observadas apenas as temperaturas de superfície de continentes, o aumento em 2016 chega a $1,4357^{\circ} \mathrm{C}$ (cerca de $53 \%$ a mais do que o incremento global).

Ainda com relação às alterações do clima global, o setor de Administração Nacional Oceânica e Atmosférica (NOAA) do Departamento de Comércio do governo dos Estados Unidos afirma que 2016 foi o ano mais quente desde o início dos registros oficiais, em 1880, sendo o terceiro ano consecutivo a estabelecer recorde de temperatura e a $15^{\mathrm{a}}$ quebra de recorde desde o início do século XXI. Esses dados evidenciam e corroboram a clara tendência de elevação das temperaturas em nível mundial ${ }^{20} \mathrm{e}$, consequentemente, de aumento dos riscos da exposição ao calor para os trabalhadores que desenvolvem suas atividades a céu aberto. $\mathrm{O}$ ano de 2017, apesar de não apresentar novo recorde, tem dados que confirmam a tendência de elevação das temperaturas, pois, em relação à série histórica registrada desde 1880 , esse ano só apresenta anomalia inferior a 2015 e $2016^{21}$.

Nesse cenário, faz-se importante dimensionar o risco da exposição ao calor a que os trabalhadores que ficam a céu aberto estão sujeitos no território brasileiro, visando oferecer subsídios para discutir a efetividade das medidas de controle praticadas e dos parâmetros e conceitos considerados admissíveis para a preservação da saúde do trabalhador. Assim, este estudo teve por objetivo identificar e mapear a influência da intensidade do trabalho no potencial de extrapolação de limites de sobrecarga térmica para trabalhadores em atividade a céu aberto em áreas rurais do Nordeste do Brasil, de modo a oferecer subsídios para a discussão de ações de prevenção.

\section{Métodos}

O instrumento utilizado para medir o potencial de exposição ao calor ambiental foi o software Sobrecarga Térmica ${ }^{16}$, desenvolvido por pesquisadores da Fundação Jorge Duprat Figueiredo de Segurança e Medicina do Trabalho (Fundacentro). O software tem sido utilizado para consultas técnicas e acompanhamentos locais de rotina, e também como ferramenta de pesquisa em trabalhos científicos ${ }^{22,23}$, dissertações ${ }^{24}$, teses e outros trabalhos acadêmicos.

O software é de livre acesso ${ }^{\mathrm{d}}$ e estima o IBUTG até a hora mais recente, entre $9 \mathrm{~h}$ e $16 \mathrm{~h}$, em qualquer ponto do território nacional que esteja a até $80 \mathrm{~km}$ de distância de uma ou mais estações meteorológicas da rede de estações automáticas do Inmet. A rede de estações automáticas dentro do território brasileiro pode ser espacialmente visualizada no endereço eletrônico do Instituto ${ }^{\mathrm{e}}$. O IBUTG é dado por:

$$
\begin{aligned}
& \text { IBUTG }=(0,7 \mathrm{Tn})+(0,3 \mathrm{Tg}) \text { [Equação 1] } \\
& \text { IBUTG }=(0,7 \mathrm{Tn})+(0,1 \mathrm{~T})+(0,2 \mathrm{Tg}) \text { [Equação 2] }
\end{aligned}
$$

d Disponível em: http://www.fundacentro.gov.br/sobrecarga-termica/inicio.

e Disponível em: http://www.inmet.gov.br/portal/index.php?r=estacoes/estacoesautomaticas. 
em que Tn é a temperatura de bulbo úmido "natural", Tg é a temperatura de globo e T é a temperatura do ar. A Equação 1 é utilizada para ambientes internos ou externos sem carga solar, e a Equação 2 para ambientes externos com carga solar.

Para a medir as variáveis meteorológicas e estimar o IBUTG, o software assume um padrão médio ponderado pela(s) distância(s) entre o ponto geográfico de interesse e a(s) estação(ões) meteorológica(s) existentes num raio de $80 \mathrm{~km}$.

Optou-se por desenvolver um estudo piloto de aplicação do software em regiões extensas e com potencial de extrapolação dos limites de sobrecarga térmica em parcela expressiva da jornada de trabalho, de modo que foi escolhida a região Nordeste do Brasil, onde o calor intenso é notório. Decidiu-se privilegiar ambientes predominantemente rurais e não urbanos devido à inviabilidade de estimar, pelo software, as diversas configurações possíveis de ambiente urbano.

A partir do mapa da região, determinou-se uma malha de pontos distantes entre si em $1^{\circ}$ de latitude e $1^{\circ}$ de longitude, isto é, as distâncias entre os pontos são de aproximadamente $110 \mathrm{~km}$. Os pontos definidos podem ser observados na Figura 1.

O software foi utilizado para determinar uma série temporal de valores do IBUTG para os pontos e período definidos. Cada um dos 132 pontos do mapa foi inserido no software Sobrecarga Térmica, gerando os valores do IBUTG de hora em hora, das $9 \mathrm{~h}$ às $16 \mathrm{~h}$, no período de $1^{\circ}$ de setembro de 2016 a 31 de agosto de 2017. Com esses dados de entrada, o software tinha um potencial de gerar 2.920 estimativas do IBUTG por ponto, ou 385.440 , no total.

A partir da série temporal das medidas realizadas, pode-se analisar a frequência com que os limites de exposição ao calor ambiental determinados na regulamentação então vigente foram ultrapassados em cada ponto geográfico de interesse. Com isso, pode-se criar uma escala de classificação do nível de risco para sobrecarga térmica dos locais avaliados.

\section{Resultados}

Dos 132 pontos geográficos referenciais definidos, 26 não apresentaram estimativas do IBUTG (pontos $\mathrm{n}^{\circ} 6,7,9,10,12,14,20,21,24,26,32$, $33,34,42,46,71,80,84,85,96,101,112,117$, 121, 152 e 128), seja por estarem a uma distância superior a $80 \mathrm{~km}$ das estações meteorológicas ou por problemas na geração de dados de algumas dessas estações. A Figura 2 mostra, para cada ponto, o percentual de estimativas em que o valor do IBUTG extrapola o limite de exposição para trabalho contínuo vigente no Anexo $\mathrm{n}^{\circ} 3$ da NR $15^{10}$ para os 3 tipos de atividades determinados pela norma: pesado, moderado e leve.

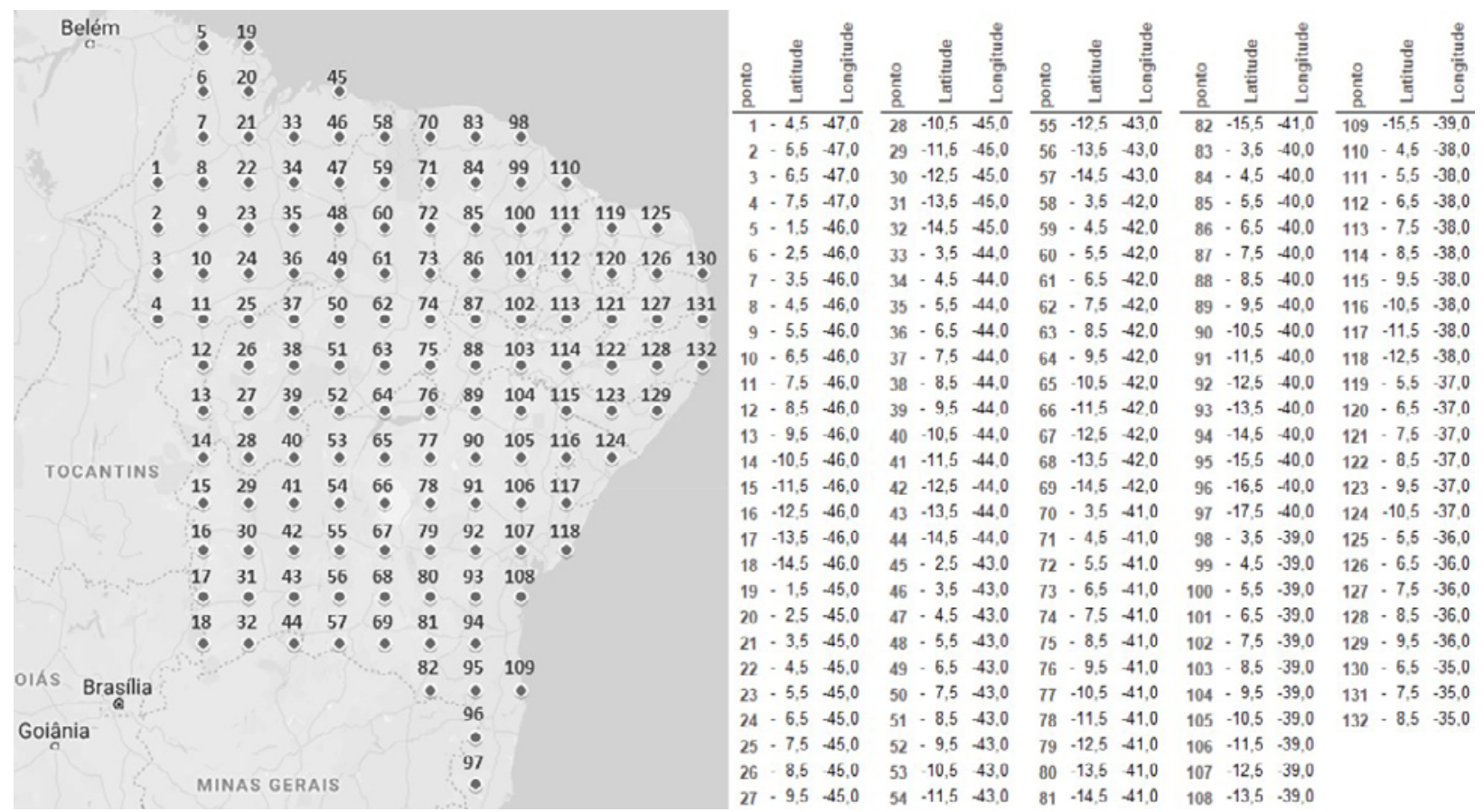

Figura 1 Pontos da região Nordeste utilizados para estimativas de valores de série temporal do IBUTG para caracterizaFonte: Adaptado de Google Maps. ção de risco de sobrecarga térmica 
Medidas de IBUTG para cada hora da jornada com limite de exposição extrapolado para trabalho contínuo do tipo pesado

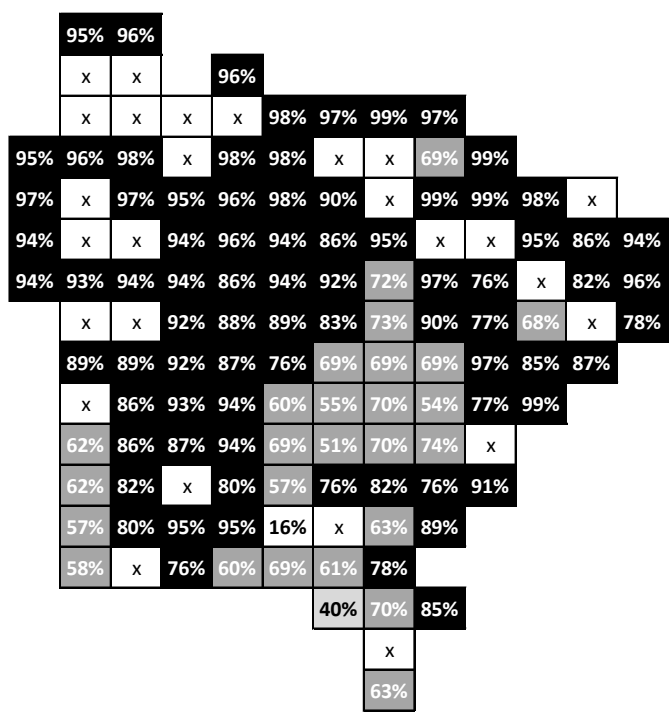

Medidas de IBUTG para cada hora da jornada com limite de exposição extrapolado para trabalho contínuo do tipo leve

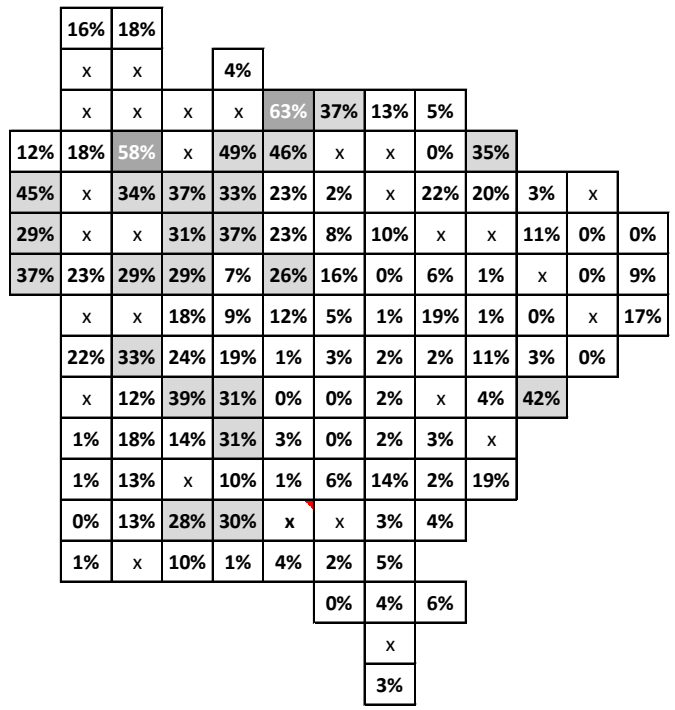

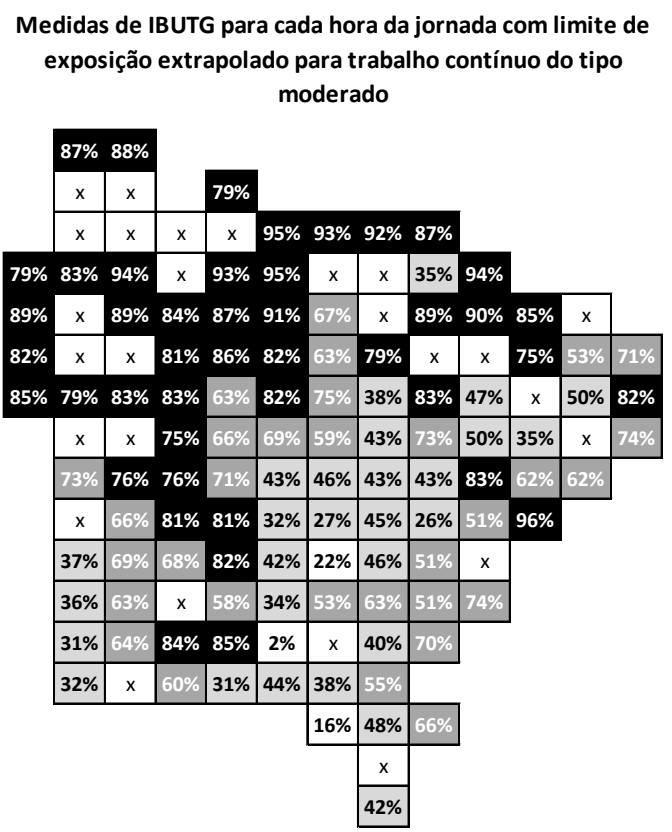

Medidas de IBUTG para cada hora da jornada com limite de moderado

Figura 2 Distribuição territorial dos valores do IBUTG que extrapolam os limites de exposição definidos pela NR $15^{10}$ para trabalho contínuo, por tipo de atividade, na região Nordeste, no período de $1^{\circ}$ de setembro de 2016 a 31 de agosto de 2017

A NR $15^{10}$ determina que, quando ultrapassado o limite para trabalho contínuo definido para cada um dos tipos de atividade (leve, moderado e pesado), deve-se adotar o regime de trabalho com descanso, podendo ser na proporção de 15, 30 ou 45 minutos de descanso a cada hora de trabalho, o que não é permitido sem a adoção de medidas adequadas de controle. Para este último caso, considerando-se que não se adotem tais medidas, será considerada a hora toda como parada (ou de descanso). O detalhamento dos limites do IBUTG para cada tipo de atividade e de seus respectivos regimes de trabalho/descanso encontra-se no Quadro ${ }^{0} 1$ do Anexo n 3 da NR15 ${ }^{10}$.

A Figura 3 mostra, percentualmente, a estimativa de quantidade de horas paradas relativas à aplicação dos regimes de trabalho/descanso acima descritos (paradas ou descanso de 15, 30 ou 45 minutos, ou ainda interrupção total das atividades em cada hora da jornada de trabalho), conforme cada tipo de atividade, no período de 12 meses estudado. 
Quantidade percentual de horas paradas relativas à aplicação dos regimes de trabalho/descanso para atividade do tipo pesado
Quantidade percentual de horas paradas relativas à aplicação dos regimes de trabalho/descanso para atividade do tipo moderado

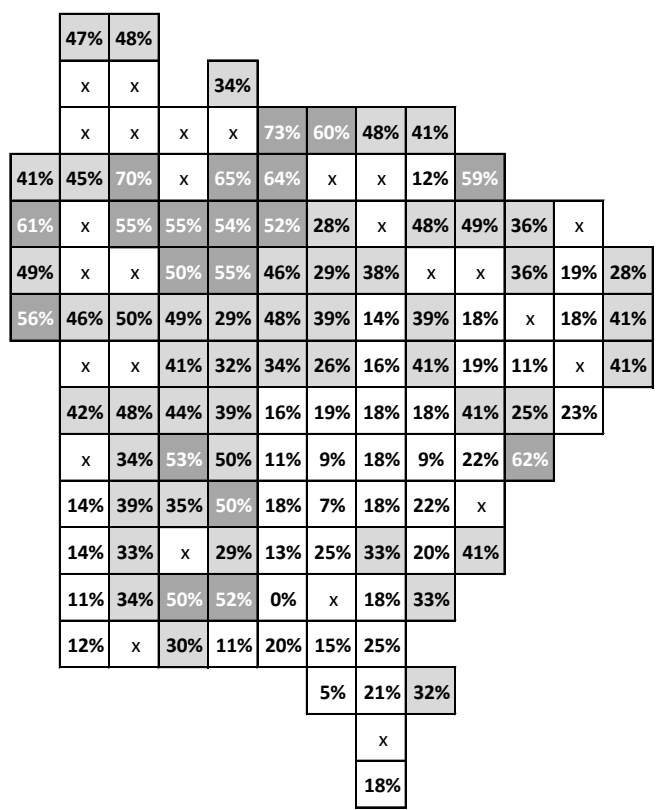

Quantidade percentual de horas paradas relativas à aplicação dos regimes de trabalho/descanso para atividade do tipo leve

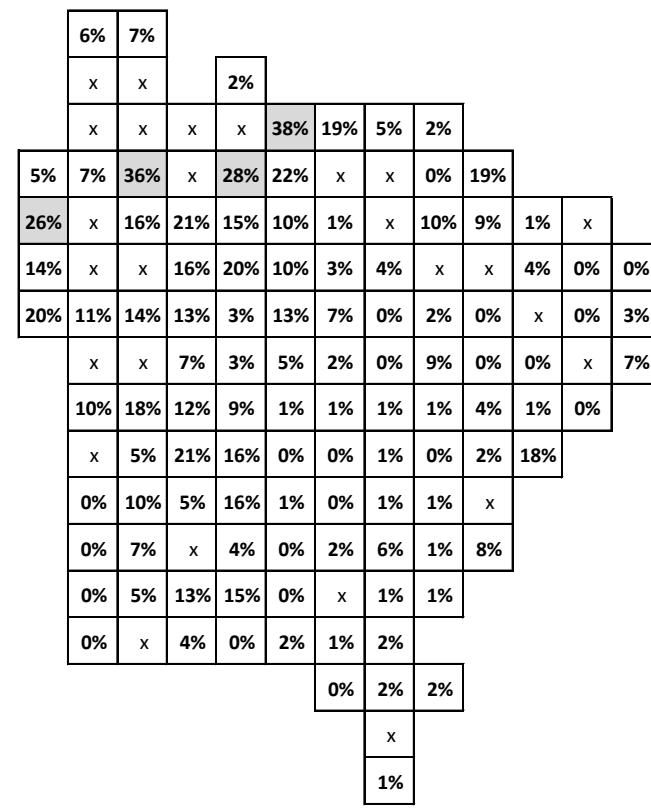

Legenda:

\% de horas paradas pela aplicação dos regimes de trabalho/descanso previstos na NR15

\begin{tabular}{|c|c|}
\hline$\%$ & $\geq 0$ e $\leq 25 \%$ \\
\hline$\%$ & $>25 \%$ e $\leq 50 \%$ \\
\hline$\%$ & $>50 \%$ e $\leq 75 \%$ \\
\hline$\%$ & $>75 \%$ \\
\hline $\mathbf{x}$ & Ponto \\
\hline
\end{tabular}

Figura 3 Horas paradas que deveriam ser cumpridas, conforme aplicação dos regimes de trabalho previstos na NR15 ${ }^{10}$, em função dos valores do IBUTG estimados para cada ponto, na região Nordeste, no período de $1^{\circ}$ de setembro de 2016 a 31 de agosto de 2017

Na Tabela 1 observa-se que, em atividades pesadas, há localidades onde o cumprimento da norma definiria paradas em mais de $80 \%$ do tempo de trabalho. Considerando o todo da região Nordeste, esse total ultrapassaria $50 \%$ de todo o período de trabalho. 
Tabela 1 Síntese dos resultados da aplicação do regime trabalho/descanso previsto na NR15 ${ }^{10}$, Anexo no 3 , segundo os valores do IBUTG estimados pelo software Sobrecarga Térmica, na região Nordeste do Brasil, no período de $1^{\circ}$ de setembro de 2016 a 31 de agosto de 2017

\begin{tabular}{lccc}
\hline & & Tipo de atividade & Leve \\
\cline { 2 - 4 } & Pesado & Moderado & 18.055 \\
\hline Horas de parada & 148.623 & 92.081 & $6,6 \%$ \\
Total de horas paradas com relação a & $54 \%$ & $33,5 \%$ & $37,5 \%$ \\
todos os pontos com dados & & $73,2 \%$ & $0 \%$ \\
Maior total de horas paradas (ponto 58) & $86,6 \%$ & $0,4 \%$ & \\
Menor total de horas paradas (ponto 68) & $5,4 \%$ & & \\
\hline
\end{tabular}

\section{Discussão e conclusão}

Os resultados obtidos a partir da aplicação do software Sobrecarga Térmica possibilitaram o mapeamento da exposição potencial ao calor em trabalhos a céu aberto na região Nordeste e permitiram observar que a diminuição da intensidade de trabalho resultaria em redução expressiva dos índices de extrapolação dos limites de sobrecarga térmica estabelecidos pela NR15. Nos trabalhos com atividades de tipo pesado, em ao menos 64 pontos geográficos (48\% dos 132 investigados) as horas de parada necessárias por extrapolação dos limites de exposição somariam 54\% do tempo analisado. A ação de diminuir o ritmo de trabalho para o nível moderado reduziria o número de pontos com limites extrapolados para 19 localidades (14\% dos 132 pontos). A introdução de pausas no trabalho é indicada como medida prática de prevenção do estresse térmico também no cenário internacional, como divulgam a European Agency for Safety and Health at Work (EU-OSHA), da União Europeia ${ }^{25}$, e o Health and Safety Executive (HSE) ${ }^{26}$, do Reino Unido, assim como o National Institute for Occupational Safety and Health (NIOSH) ${ }^{27}$, dos Estados Unidos, preconiza a redução do ritmo de trabalho.

No entanto, uma proposta de intervenção para redução do ritmo/intensidade de trabalho para o tipo de atividade leve não faria sentido, pois dificilmente se conseguiria enquadrar tarefas como plantio e colheita nessa categoria, que é descrita na norma por ações como: "Sentado, movimentos moderados com braços e tronco (ex.: datilografia). [...] De pé, trabalho leve, em máquina ou bancada, principalmente com os braços"10.

O fato de os dados mostrarem que, para a região Nordeste, em mais de 50\% do tempo deveriam ocorrer paradas - devido às condições climáticas de calor que excedem os limites legais - tornaria irrefutável a necessidade de monitoramento ambiental praticamente constante das atividades de trabalho a céu aberto na região. Contudo, sabe-se que o monitoramento in loco, na prática, é de difícil execução por diversas razões, como o elevado custo dos equipamentos e a baixa qualidade de equipamentos nacionais e similares de menor valor; a indisponibilidade de pessoal qualificado para realizar as medições; o acompanhamento esporádico das atividades de trabalho; e a falta de autonomia do técnico de segurança para suspender as atividades dos trabalhadores. Assim sendo, em vez de se tentar investir no monitoramento - que, além de ser praticamente inviável, é inócuo, por si só, para preservar a saúde dos trabalhadores -, seria mais viável e, consequentemente, mais efetiva a adoção de políticas de prevenção.

Uma possibilidade seria garantir um ritmo de trabalho de menor intensidade, proibindo, por exemplo, a prática comum de atrelar o pagamento à produção. Essa ação pode ser eficaz tanto para a melhoria das condições de trabalho como para o atendimento à legislação. A despeito disso, há uma grande dificuldade para a aplicação prática dessa medida, pois se encontra resistência tanto por parte das empresas, que visam à otimização dos resultados de lucro e produtividade, como dos trabalhadores, que se focam pontualmente no aumento de seus rendimentos. No entanto, diante das condições climáticas adversas para atividades de trabalho de alta intensidade em regiões como a aqui estudada, a discussão de outras formas de remuneração que possam satisfazer trabalhadores e empresas se mostra fundamental.

De qualquer forma, para os trabalhadores que desenvolvem suas atividades a céu aberto em ambientes predominantemente rurais, em locais classificados como de alto risco pelo histórico climático-ambiental, é indicada, de antemão, a adoção de medidas de prevenção, uma vez que, se fosse realizado o monitoramento em tempo integral, os resultados revelariam a necessidade, em grande parte, senão em todo o tempo, de tais medidas.

Em suma, há muito a ser feito para concretizar medidas efetivas que garantam melhores condições de preservação da saúde do trabalhador quanto à exposição ao calor, sobretudo as relacionadas à redução da intensificação do trabalho. 


\section{Contribuições de autoria}

Roscani RC e Maia PA elaboraram e delinearam o estudo e fizeram o levantamento de dados. Juntamente com Monteiro MI, analisaram e interpretaram os dados, elaboraram o manuscrito e fizeram sua revisão crítica. Todos participaram da aprovação final da versão publicada e se responsabilizam publicamente por seu conteúdo.

\section{Referências}

1. Todd N, Valleron AJ. Space-time covariation of mortality with temperature: a systematic study of deaths in France, 1968-2009. Environ Health Perspect [Internet]. 2015 [citado em 16 out 2019];123(7):659-64. Disponível em: https://doi. org/10.1289/ehp.1307771

2. Basu R, Samet JM. Relation between elevated ambient temperature and mortality: a review of the epidemiologic evidence. Epidemiol Rev [Internet]. 2002 [citado em 16 out 2019];24(2):190202. Disponível em: https://doi.org/10.1093/epirev/ mxf007

3. Xiang J, Bi P, Pisaniello D, Hansen A. Health impacts of workplace heat exposure: an epidemiological review. Ind Health. 2014;52(2):91101.

4. Tawatsupa B, Lim LL, Kjellstrom T, Seubsman SA, Sleigh A; Thai Cohort Study Team. Association between occupational heat stress and kidney disease among 37,816 workers in the Thai Cohort Study (TCS). J Epidemiol [Internet]. 2012 [citado em 30 maio 2014];22(3):251-60. Disponível em: http://www.ncbi.nlm.nih.gov/pubmed/22343327

5. Barbosa CMG. Avaliação cardiovascular e respiratória em um grupo de trabalhadores cortadores de cana-de-açúcar queimada no estado de São Paulo [tese]. São Paulo: Universidade de São Paulo; 2011. 144 p.

6. Barbosa CMG, Terra-Filho M, Albuquerque ALP, Di Giorgi D, Grupi C, Negrão CE, et al. Burnt sugarcane harvesting: cardiovascular effects on a group of healthy workers, Brazil. PLoS One [Internet]. 2012 [citado em 8 out 2014];7(9):e46142. Disponível em: http://www.ncbi.nlm.nih.gov/pmc/ articles/PMC3459900

7. Bethel JW, Harger R. Heat-related illness among Oregon farmworkers. Int J Environ Res Public Health [Internet]. 2014 [citado em 29 set 2014];11(9):9273-85. Disponível em: http://www. ncbi.nlm.nih.gov/pmc/articles/PMC4199019/pdf/ ijerph-11-09273.pdf

8. Mirabelli MC, Richardson DB. Heat-related fatalities in North Carolina. Am J Public Health [Internet]. 2005 [citado em 18 jun 2015];95(4):6357. Disponível em: http://ajph.aphapublications.org/ doi/pdf/10.2105/AJPH.2004.042630

9. Jay O, Kenny GP. Heat exposure in the Canadian workplace. Am J Ind Med [Internet]. 2010 [citado em 17 out 2019];53(8):842-53. Disponível em: https://doi.org/10.1002/ajim.20827
10. Brasil, Ministério do Trabalho. Portaria MTb $\mathrm{n}^{\mathrm{O}} 3.214$, de 8 de junho de 1978. Diário Oficial da União. 6 jul 1978 [citado em 24 out 2019];1. Disponível em: https://enit.trabalho.gov.br/portal/ images/Arquivos_SST/SST_NR/NR-15.pdf

11. Yaglou CP, Minard D. Control of heat casualties at military training centers. AMA Arch Ind Health. 1957;16(4):302-16.

12. International Organization for Standardization. ISO 7243:1989: hot environments: estimation of the heat stress on working man, based on the WBGT-index (wet bulb globe temperature). Geneva; 1989.

13. Crowe J, Moya-Bonilla JM, Román-Solano B, Robles-Ramírez A. Heat exposure in sugarcane workers in Costa Rica during the non-harvest season. Glob Health Action [Internet]. 2010 [citado em 17 out 2019];3(1):5619. Disponível em: https:// doi.org/10.3402/gha.v3i0.5619

14. Kjellstrom T, Lemke B, Otto M. Mapping occupational heat exposure and effects in South-East Asia: ongoing time trends 19802011 and future estimates to 2050. Ind Health [Internet]. 2013 [citado em 17 out 2019];51(1):5667. Disponível em: https://doi.org/10.2486/ indhealth.2012-0174

15. Brasil, Ministério do Trabalho e Emprego. Portaria SSST $n^{\circ} 25$, de 29 de dezembro de 1994. Diário Oficial da União [Internet]. $30 \mathrm{dez} 1996$ [citado em 16 out 2019]. Disponível em: https://www.legisweb. com.br/legislacao/?id=181316

16. Maia PA, Ruas ÁC, Bitencourt DP. Wet-bulb globe temperature index estimation using meteorological data from São Paulo State, Brazil. Int J Biometeorol [Internet]. 2015 [citado em $12 \mathrm{fev}$ 2015];59(10):1395-403. Disponível em: http://www. ncbi.nlm.nih.gov/pubmed/25634645

17. Alves F. Por que morrem os cortadores de cana? Saude Soc. 2006;15(3):90-8.

18. Vilela RAG, Laat EF, Luz VG, Silva AJN, Takahashi, MAC. Pressão por produção e produção de riscos: a "maratona" perigosa do corte manual da canade-açúcar. Rev Bras Saude Ocup [Internet]. 2015 [citado em 10 set 2015];40(131):30-48. Disponível em: http://www.scielo.br/scielo.php?script =sci arttext\&pid $=$ S0303-76572015000100030\&lng $=$ en. http://dx.doi.org/10.1590/0303-7657000075413.

19. Novo recorde climático coloca mundo em "território verdadeiramente inexplorado", alerta 
ONU. ONU Brasil [Internet]. 22 mar 2017 [citado em 28 mar 2017]. Disponível em: https:// nacoesunidas.org/novo-recorde-climaticocoloca-mundo-em-territorio-verdadeiramenteinexplorado-alerta-onu/

20. NOAA National Centers for Environmental Information. Climate at a glance: global time series [Internet]. Asheville; 2017 [citado em 28 mar 2017]. Disponível em: https://www.ncdc.noaa.gov/ cag/time-series/global/globe/landean/ytd/12/18802017

21. NOAA National Centers for Environmental Information. Climate at a glance: global time series [Internet]. Asheville; 2018 [citado em 16 fev 2018]. Disponível em: https://www.ncdc.noaa.gov/cag/ time-series/global/globe/land/ytd/12/1880-2017

22. Roscani RC, Bitencourt DP, Maia PA, Ruas AC. Risco de exposição à sobrecarga térmica para trabalhadores da cultura de cana-de-açúcar no estado de São Paulo, Brasil. Cad Saude Publica [Internet]. 2017 [citado em 14 out 2019];33(3):e00211415. Disponível em: http:// dx.doi.org/10.1590/0102-311x00211415

23. Amorim A, Labaki L, Maia P, Barros T, Marcelo A. Occupational exposure to heat in outdoor activities in building constructions in Southeastern Brazil.
In: Arezes PM, organizador. Advances in safety management and human factors. New York: Springer; 2018. p. 393-403.

24. Roscani, RC. Risco de exposição à sobrecarga térmica para trabalhadores da cultura de cana-deaçúcar no estado de São Paulo [dissertação]. São Paulo: Fundacentro; 2015. 55 p.

25. European Agency for Safety and Health at Work. Prevention tips: heat stress at work [Internet]. Bilbao: EU-OSHA; 2015 [citado em 14 out 2019]. Disponível em: https:/osha.europa.eu/en/themes/ dangerous-substances/practical-tools-dangeroussubstances/prevention-tips-heat-stress-work

26. Health and Safety Executive. Employee's guide: temperature [Internet]. Bootle: HSE; 2015 [citado em 14 out 2019]. Disponível em: http://www.hse. gov.uk/temperature/thermal/workers.htm

27. Jacklitsch B, Williams WJ, Musolin K, Coca A, Kim J-H, Turner N. Occupational exposure to heat and hot environments: revised criteria 2016: criteria for a recommended standard occupational [Internet]. Cincinnati: NIOSH; 2016 [citado em 14 out 2019]. Disponível em: https://www.cdc.gov/niosh/ docs/2016-106/pdfs/2016-106.pdf?id=10.26616/ NIOSHPUB2016106 